\title{
Long-Term Clinical Outcomes of Endoscopic Submucosal Dissection in Patients with Early Gastric Cancer: A Prospective Multicenter Cohort Study
}

\author{
Sang Gyun Kim ${ }^{1}$, Chan Mi Park², Na Rae Lee ${ }^{2}$, Jiyoung Kim² ${ }^{2}$ Da Hyun Lyu², Seung-Hee Park², II Ju Choi ${ }^{3}$, Wan Sik Lee', \\ Seun Ja Park ${ }^{5}$, Jae Jun Kim${ }^{6}$, Ji Hyun Kim${ }^{7}$, Chul-Hyun Lim ${ }^{8}$, Joo Young Cho ${ }^{9}$, Gwang Ha Kim ${ }^{10}$, Yong Chan Lee ${ }^{11}$, \\ Hwoon-Yong Jung ${ }^{12}$, Jun Haeng Lee $^{6}$, Hoon Jai Chun ${ }^{13}$, and Sang-Yong Seol ${ }^{7}$ \\ ${ }^{\prime}$ Department of Internal Medicine and Liver Research Institute, Seoul National University, ${ }^{2}$ National Evidence-Based Healthcare Collaborating \\ Agency, Seoul, ${ }^{3}$ Center for Gastric Cancer, National Cancer Center, Goyang, ${ }^{4}$ Department of Internal Medicine, Chonnam National University \\ Hwasun Hospital, Hwasun, ${ }^{5}$ Department of Internal Medicine, Kosin University College of Medicine, Busan, ${ }^{6}$ Department of Medicine, Samsung \\ Medical Center, Sungkyunkwan University School of Medicine, Seoul, ${ }^{7}$ Department of Internal Medicine, Inje University Busan Paik Hospital, \\ Busan, ${ }^{8}$ Department of Internal Medicine, College of Medicine, The Catholic University of Korea, ${ }^{9}$ Digestive Disease Center, Soonchunhyang \\ University Hospital, Seoul, ${ }^{10}$ Department of Internal Medicine, Pusan National University School of Medicine, Busan, ${ }^{11}$ Department of Internal \\ Medicine, Yonsei University College of Medicine, ${ }^{12}$ Department of Gastroenterology, Asan Medical Center, University of Ulsan College of \\ Medicine, and ${ }^{13}$ Department of Internal Medicine, Institute of Digestive Diseases and Nutrition, Korea University College of Medicine, Seoul, \\ Korea
}

See editorial on page 371

Background/Aims: Endoscopic submucosal dissection (ESD) has been regarded as a curative treatment for early gastric cancer (EGC) in indicated cases. The aim of this study was to evaluate the nationwide long-term clinical outcomes of ESD for EGC in Korea. Methods: A prospective multicenter cohort study was performed to evaluate the long-term efficacy of ESD for EGC within pre-defined indications at 12 institutes in Korea. The cases that met the expanded criteria upon pathological review after ESD were followed for 5 years. The primary outcome was 5-year disease specific free survival. Results: Six hundred ninety-seven patients with 722 EGCs treated with ESD were prospectively enrolled and followed for 5 years. Complete resection was achieved in $81.3 \%$ of the cases, and curative resection was achieved in $86.1 \%$. During the 5 -year follow-up, the overall survival rate was $96.6 \%$, and the disease specific free survival rate was $90.6 \%$. Local recurrence developed in 0.9\%, and metachronous tumor development occurred in $7.8 \%$; both conditions were treated by endoscopic or surgical treatment. Distant metastasis developed in $0.5 \%$ during follow-up. Conclusions: ESD showed excellent long-term clinical outcomes and can be accepted as a curative treatment for patients with EGC who meet the expanded criteria in final pathology studies.
(Gut Liver 2018;12:402-410)

Key Words: Survival; Endoscopic mucosal dissection; Stomach neoplasms

\section{INTRODUCTION}

Gastric cancer is the fourth most common malignancy worldwide, and the second cause among all malignancy in Korea. ${ }^{1,2}$ As the Korean National Cancer Screening Program has provided the population over 40 years screening upper gastrointestinal endoscopy for gastric cancer every 2 years, the rate of detection in early stage of gastric cancer has increased up to 70\% in Korea. ${ }^{3,4}$ Increased proportion of early detection of cancer and the progress of endoscopic techniques and accessories has replaced surgical resection with endoscopic resection for early gastric cancer (EGC) with indication of negligible risk of lymph node or distant metastasis.

Endoscopic submucosal dissection (ESD) has been accepted as a curative treatment modality for EGC in the indicated case, and shown many advantages in terms of quality of life and short and long-term clinical outcomes. ${ }^{5-7}$ The short-term clinical outcomes has shown excellent en-bloc and curative resection rate around 90\%, and minimal risk of adverse events such as bleeding and perforation. ${ }^{6}$ Quality of life did not deteriorate immediately after ESD for EGC, and improved more significantly

Correspondence to: Sang-Yong Seol

Department of Internal Medicine, Inje University Busan Paik Hospital, 75 Bokji-ro, Busanjin-gu, Busan 47392, Korea

Tel: +82-51-890-6158, Fax: +82-51-891-6438, E-mail: seolsymd@hanmail.net

Received on September 18, 2017. Revised on November 2, 2017. Accepted on November 24, 2017. Published online March 29, 2018

pISSN 1976-2283 eISSN 2005-1212 https://doi.org/10.5009/gnl17414

Sang Gyun Kim and Chan Mi Park contributed equally to this work as first authors.

(a) This is an Open Access article distributed under the terms of the Creative Commons Attribution Non-Commercial License (http://creativecommons.org/licenses/by-nc/4.0) which permits unrestricted non-commercial use, distribution, and reproduction in any medium, provided the original work is properly cited. 
at up to 6 months without significant complications. ${ }^{5}$

Although the long-term clinical outcomes have been reported to be excellent in the cases within absolute or expanded criteria, the evidence from nationwide large-scaled prospective cohort study has not been yet fully clarified..$^{7-9}$ Moreover, the long-term clinical outcomes can be influenced by the enrolled criteria of ESD, and the pathological diagnosis which decides the criteria of ESD may be changed by inter-observer variations. ${ }^{10}$ Therefore, unified pathological diagnosis and the criteria of enrollment are indispensable to evaluate the long-term clinical outcomes such as survival of ESD for EGC.

In this study, nationwide large-scaled prospective cohort has been followed to evaluate the long-term clinical outcomes of ESD for EGC with a central pathological review.

\section{MATERIALS AND METHODS}

\section{Study design}

This study was planned to evaluate the long-term clinical outcomes of ESD for EGC for 5 years in Korea by a nationwide, multicenter, and prospective cohort manner. The patients who were diagnosed as EGC in indicated case and planned to undergo ESD were prospectively enrolled from 12 nationwide hospitals in Korea from May 2010 to December 2011. The minimum requirement for a center participating in the study was at least 20 ESD cases per year. This study was performed in collaboration with National Evidence-based Healthcare Collaborating Agency (NECA), which is the national research agency of Korea that was established to provide authentic and quality information about medical devices, medicines, and health technology through objective and reliable analyses.

\section{Patients}

The Inclusion criteria were as follows; (1) adults aged at least 20 years, (2) gross tumor size not more than $3 \mathrm{~cm}$ in longest diameter, (3) well- or moderately differentiated adenocarcinoma or adenoma by pathological evaluation of the biopsy specimen, (4) no endoscopic ulceration, and (5) tumor expected to be confined within mucosa without evidence of lymph node or distant metastasis. The exclusion criteria were as follows; (1) history of malignancy within 5 years of enrollment, (2) history of partial gastrectomy, (3) severe co-morbidity, (4) bleeding tendency, and (5) pregnancy.

All participants were provided with written informed consents for the study, and the institutional review boards of all centers approved the study. The protocol was registered at ClinicalTrials.gov (identifier number: NCT01132469). This study had been conducted in accordance with the Declaration of Helsinki.

\section{ESD procedure}

ESD was performed under conscious sedation with midazolam and/or propofol, and under cardiopulmonary monitoring. All the procedures were performed as previously described. ${ }^{11}$ Marking was made $2 \mathrm{~mm}$ outside of the lesion using the tip of an electrosurgical device or argon plasma coagulation. Then, various submucosal solutions were injected into the submucosal layer to make a submucosal cushion, and a circumferential mucosal incision was made using various electrosurgical knives. Finally, hemostasis was performed for any oozing or exposed vessel both during and after the procedure. ${ }^{6}$

\section{Pathological evaluation}

Resected specimens were fixed in 10\% formalin and embed-
1,160 Registered number of patients

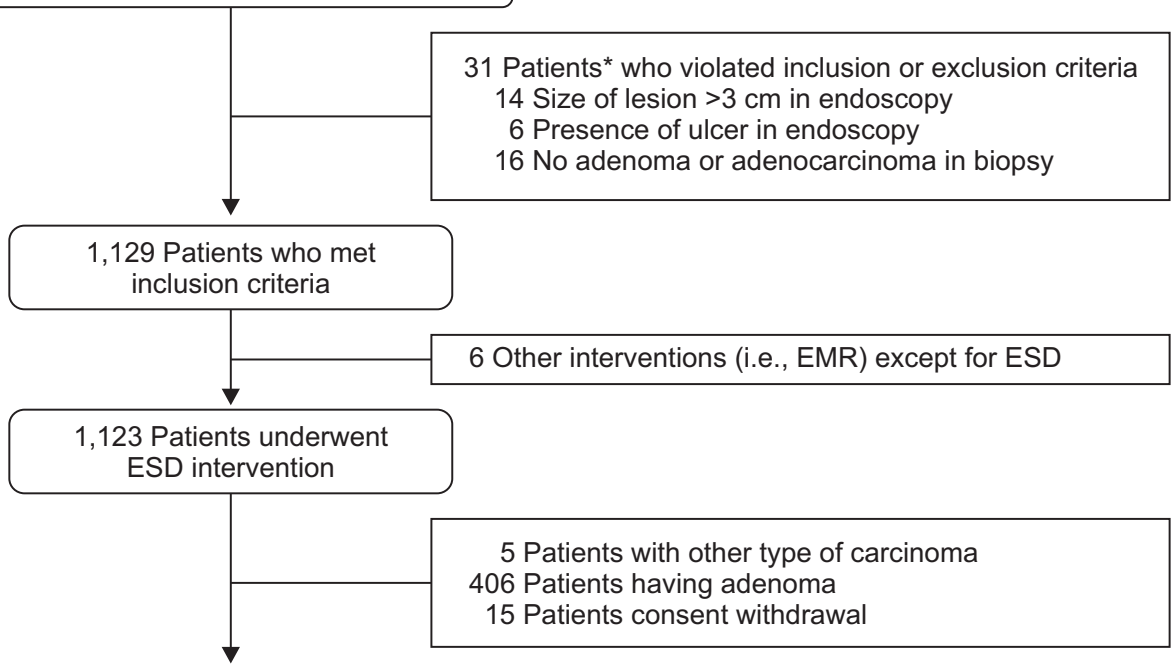

697 Patient cohort (722 adenocarcinoma lesions)
Fig. 1. Enrollment of patients. EMR, endoscopic mucosal resection; ESD, endoscopic submucosal dissection. *In 31 patients, viotation of enrollment is overlapped. 
ded into paraffin for histological evaluation. Sections were made in 2-mm thickness interval, and stained with hematoxylin and eosin. Pathological diagnoses were made according to Japanese Classification of Gastric Cancer. ${ }^{12}$ After the initial evaluation, slides of specimens were sent to an independent central pathology review board, which consisted of 16 specialists in gastrointestinal pathology who are members of the Korean Society of Pathologists, and finally diagnosed by an agreement rate of $70 \%$ or more.

En-bloc resection was defined as a resection of tumor in onepiece. Curative resection was defined as en-bloc resection with tumor-free margin within expanded criteria, and complete resection as a resection of tumor confined to mucosa with tumorfree margin in differentiated histology and without lymphovascular invasion.

Table 1. Baseline Characteristics of Patients and Lesions $(n=697)$

\begin{tabular}{|c|c|}
\hline Characteristic & Value \\
\hline Male sex & $537(77.0)$ \\
\hline Age, yr & $62.7 \pm 9.2$ \\
\hline Family history of gastric cancer & $98(14.1)$ \\
\hline Multiplicity of cancer & $24(3.4)$ \\
\hline \multicolumn{2}{|l|}{ Location } \\
\hline Lower 1/3 & $438(62.8)$ \\
\hline Middle 1/3 & $177(25.4)$ \\
\hline Upper $1 / 3$ & $82(11.8)$ \\
\hline \multicolumn{2}{|l|}{ Histology } \\
\hline Well or moderately differentiated & $677(97.1)$ \\
\hline Undifferentiated & $20(2.9)$ \\
\hline \multicolumn{2}{|l|}{ Tumor size, mm } \\
\hline$\leq 20$ & $512(73.5)$ \\
\hline $21-30$ & $123(17.6)$ \\
\hline$>30$ & $62(8.9)$ \\
\hline \multicolumn{2}{|l|}{ Depth of tumor invasion } \\
\hline Lamina propria & $350(50.2)$ \\
\hline Muscularis mucosa & $232(33.3)$ \\
\hline Submucosa & $115(16.5)$ \\
\hline \multicolumn{2}{|l|}{ Gross morphology } \\
\hline Depressed & $375(53.8)$ \\
\hline Elevated & $168(24.1)$ \\
\hline Flat & $104(14.9)$ \\
\hline Unclassified & $50(7.2)$ \\
\hline Tumor-positive lateral margin & $3(0.4)$ \\
\hline Tumor-positive vertical margin & $13(1.9)$ \\
\hline Lymphatic invasion & $31(4.5)$ \\
\hline En-bloc resection & $691(99.1)$ \\
\hline Complete resection & $567(81.3)$ \\
\hline Curative resection & $600(86.1)$ \\
\hline
\end{tabular}

Data are presented as number (\%) or mean $\pm \mathrm{SD}$.

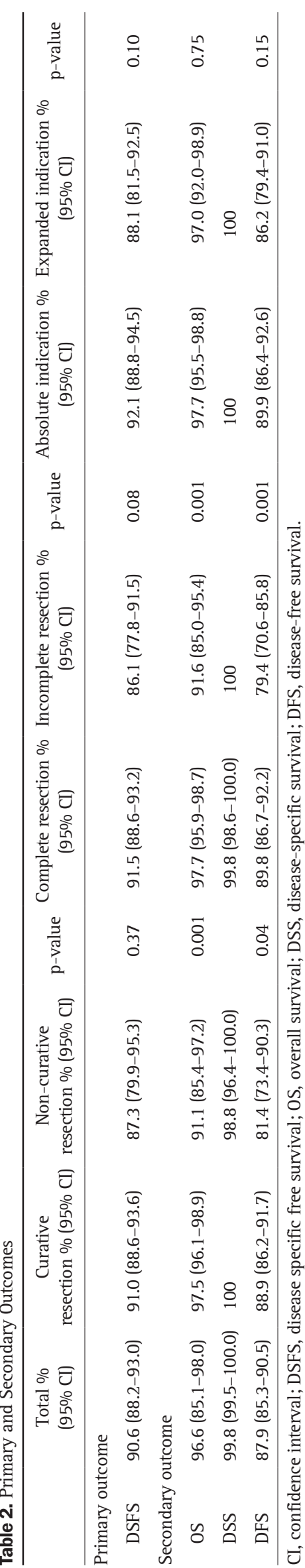


Absolute indication was defined as a resection of tumor (1) confined to mucosa, (2) with well or moderately differentiated adenocarcinoma, and (3) within $2 \mathrm{~cm}$ in diameter in final pathology. Expanded criteria was defined as a resection of tumor of (1) intramucosal differentiated adenocarcinoma without ulcer irrespective of size, (2) intramucosal differentiated adenocarcinoma with ulcer and size $\leq 3 \mathrm{~cm}$, (3) intramucosal undifferentiated histology without ulcer and size $\leq 2 \mathrm{~cm}$, or 4 ) differentiated adenocarcinoma with submucosal invasion $\leq 500 \mu \mathrm{m}$ (sm1) and size $\leq 3 \mathrm{~cm}$.

\section{Follow-up and clinical outcomes}

Periodic follow-up was performed at 3 months, 6 months, 1 year, and annually for 5 years after ESD with endoscopy, abdominal computerized tomography (CT), chest radiography, and laboratory tests. The primary outcome was 5-year disease specific free survival rates and the secondary outcomes were as follows; (1) 5-year overall survival rate, (2) 5-year disease specific survival rate, (3) 5-year disease-free survival rate, and (4)

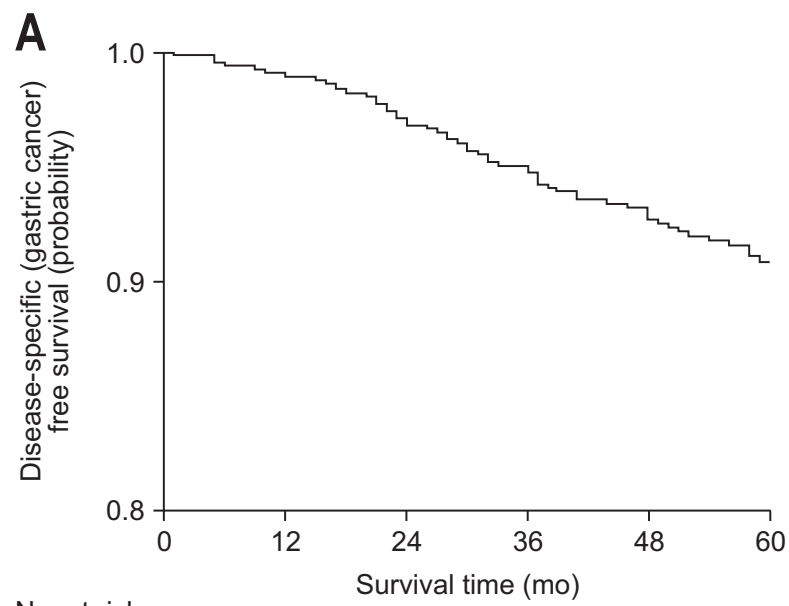

No. at risk 697 667

621

581

533

268

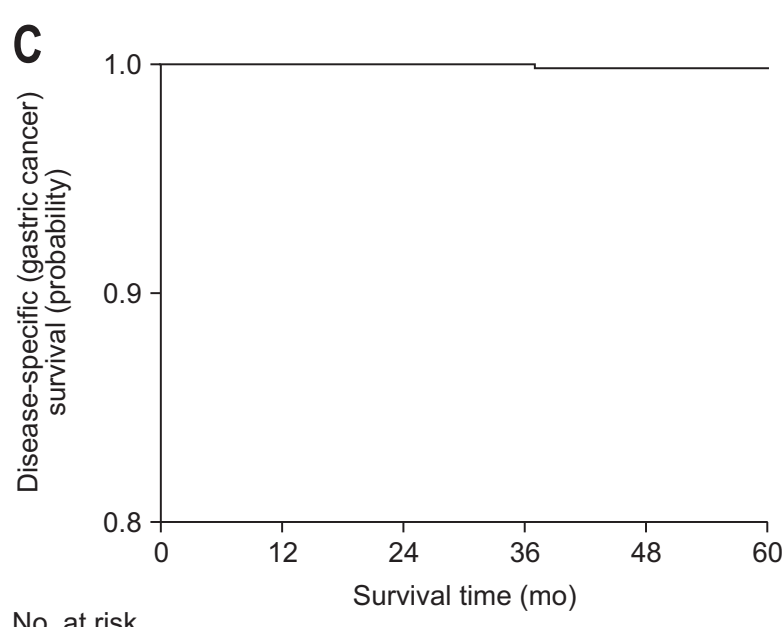

No. at risk

639

610

566

290

Fig. 2. Survival rates of all patients.

recurrence rate. Other secondary outcomes such as short-term outcomes, safety analyses, quality of life, and cost-effective analyses were reported in the previous reports. ${ }^{5,6,13}$

In this study, 5-year disease specific free survival rate was defined as a survival free from local or distant recurrence of index cancer. And, 5-year overall survival rates was defined as a survival free from all death, 5-year disease specific survival rate as a survival free from all gastric cancer-related death, 5-year disease-free survival rate as a survival free from metachronous or local/distant recurrence and/or all death, and recurrence rate as metachronous or local/distant recurrence.

Local recurrence was defined as a recurrence of index tumor at the site of ESD, and distant metastasis as a recurrence of index tumor outside the stomach. Synchronous tumor was defined as a development of new tumor at other site rather than ESD site within 1 year after ESD, and metachronous tumor as a development of new tumor beyond 1 year after ESD. Distant recurrence included distant metastasis, synchronous and metachronous tumor during follow-up.

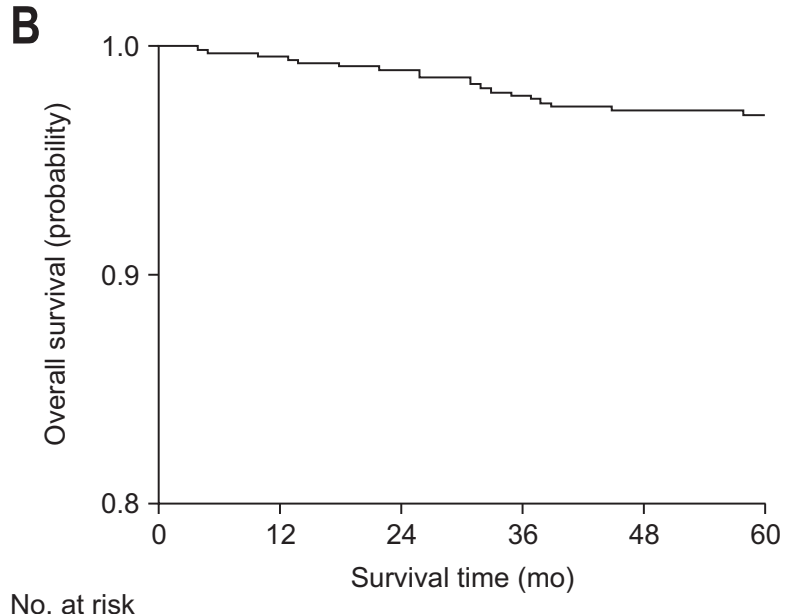

No. at risk

$\begin{array}{lllll}697 & 673 & 639 & 610 & 566\end{array}$

290

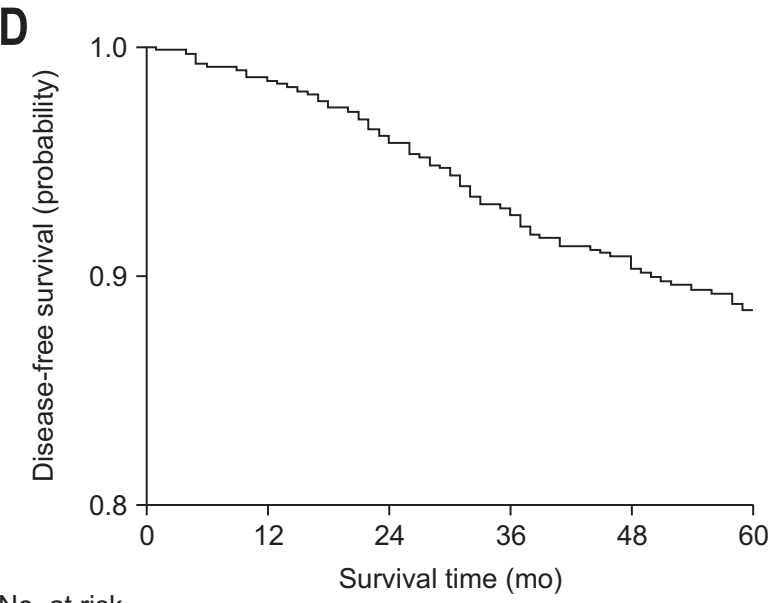

No. at risk 697 
The patients were excluded in the analysis if they received immediate additional endoscopic or surgical resection with the pathologic results beyond expanded criteria.

\section{Sample size and statistical analyses}

Sample size was calculated based on the non-inferiority test compared with the expected 5-year overall survival rate of 92.7\% with a margin of 5\%, which was obtained from historical data of surgery for EGC. ${ }^{14}$ A study enrollment of 24 months and 5-year follow-up required 521 patients with EGC for statistical analysis. Considering a drop-out rate of $10 \%$ with a power of $80 \%$ and an alpha error of 2.5\%, 572 patients with EGC were required as the final sample size.

Demographic information was presented as descriptive statistics. Survival rates were calculated using the Kaplan-Meyer method, with comparison between the groups using log-rank test. All tests of significance were two-tailed, and p-values $<0.05$ were considered statistically significant. Statistical analy-

A

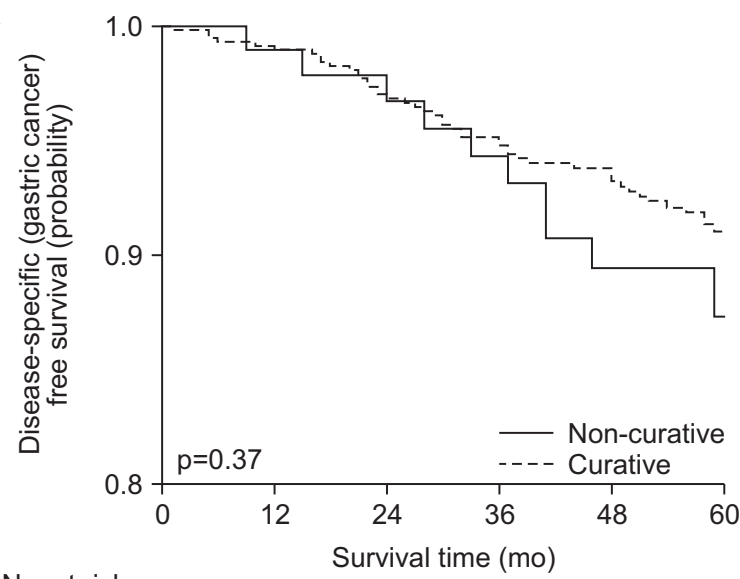

No. at risk $\begin{array}{crrrrrr}\text { Non-curative } & 97 & 93 & 85 & 79 & 71 & 29 \\ \text { Curative } & 600 & 574 & 537 & 503 & 462 & 239\end{array}$

C

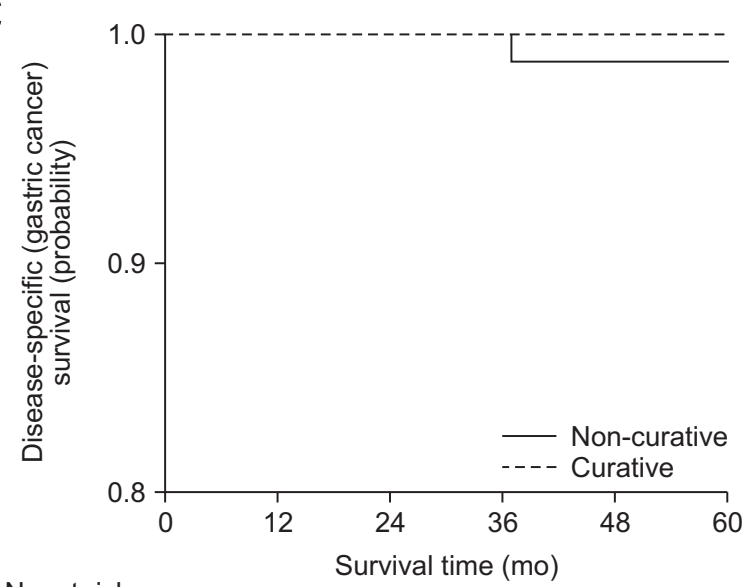

No. at risk Non-curative 97 Curative 600

94
579

86
553

\begin{abstract}
82
528
\end{abstract}
76
490

32
258 ses were conducted using SAS version 9.4 (SAS Institute Inc., Cary, NC, USA).

\section{RESULTS}

\section{Baseline characteristics of patients and lesions}

A total of 1,160 patients had been registered for screening between May 2010 and December 2011, and 722 EGC lesions identified from 697 patients were finally included in this study. Median follow-up duration was 59 months (range, 2 to 60 months) (Fig. 1).

Male patients formed $77 \%$ of the study population and the mean age was 62.7 years. Family history of gastric cancer was accompanied in $14.1 \%$ of the patients and multiple lesions were found initially in 3.4\% of the patients. The lesion was located in lower third in 62.8\% and showed differentiated histology in 97.1\%. Tumor size was not more than $3 \mathrm{~cm}$ in $91.1 \%$ and the depth of tumor invasion was confined to mucosa in 83.5\%.

B

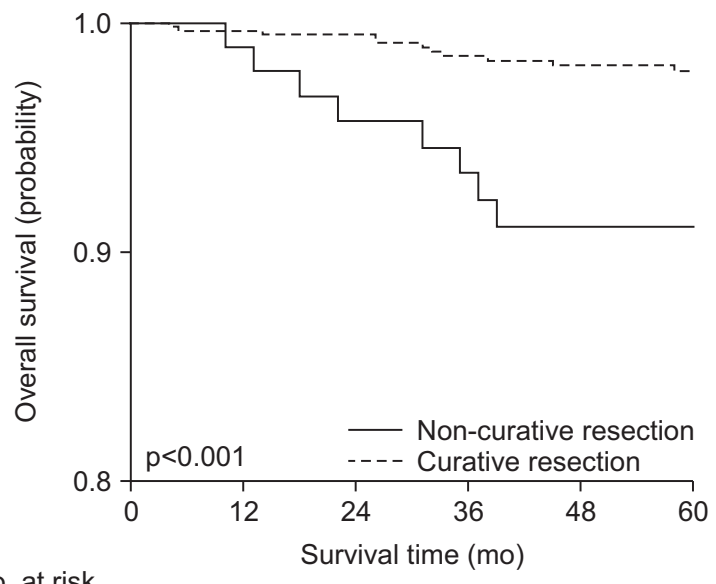

No. at risk

$\begin{array}{lrrrrrr}\text { Non-curative resection } & 97 & 94 & 86 & 82 & 76 & 32\end{array}$

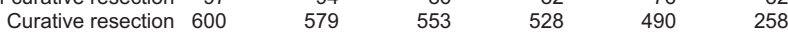

D

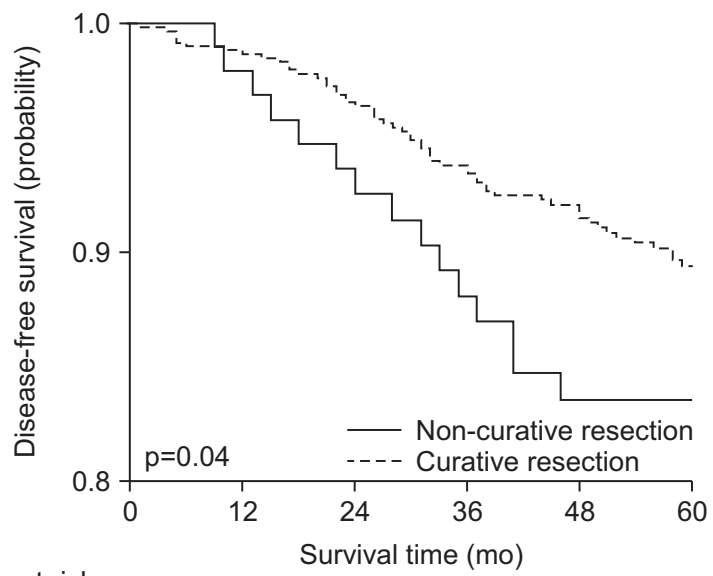

No. at risk

$\begin{array}{lrrrrrr}\text { Non-curative resection } & 97 & 93 & 85 & 79 & 71 & 29 \\ \text { Curative resection } & 600 & 574 & 536 & 502 & 462 & 239\end{array}$

Fig. 3. Survival rates according to curative resection. 
Gross type was depressed morphology in 53.8\% and lymphatic tumor invasion was revealed in $4.5 \%$. Tumor was positive in $0.4 \%$ of lateral margin and 1.9\% of vertical margin.

En-bloc resection rate was 99.1\% (691/697). Complete resection was obtained in 567 patients (81.3\%; 95\% confidence interval [CI], 78.7\% to $84.4 \%$ ), which was defined as a differentiated adenocarcinoma limited to the mucosal layer with tumorfree margins in the resected specimen. Curative resection was achieved in $86.1 \%$ (Table 1).

\section{Primary and secondary outcomes}

Five-year disease specific free survival rate as primary outcome was $90.6 \%$ (95\% CI, 88.2\% to 93.0\%). In secondary outcomes, 5-year overall survival rate was 96.6\% (95\% CI, 85.1\% to $98.0 \%$ ), 5-year disease specific survival rate was 99.8\% (95\% CI, $99.5 \%$ to $100.0 \%$ ), and 5-year disease-free survival rate was

Table 3. Recurrence Rate

\begin{tabular}{lcccc}
\hline & Total \% $(95 \% \mathrm{CI})$ & Curative resection \% (95\% CI) & Non-curative resection \% (95\% CI) & $\mathrm{p}$-value \\
\hline All recurrence & $9.0(6.9-11.8)$ & $8.9(6.6-11.8)$ & $13.0(5.2-20.2)$ & 0.72 \\
Local recurrence & $0.9(0.4-2.0)$ & $0.7(0.3-1.8)$ & $2.4(0.6-9.1)$ & 0.17 \\
Distant metastasis & $0.5(0.2-1.5)$ & $0.2(0.0-1.2)$ & $2.4(0.6-9.4)$ & 0.01 \\
Metachronous recurrence & $7.8(5.8-10.4)$ & $8.1(5.9-11.0)$ & $5.9(2.2-15.8)$ & 0.40 \\
\hline
\end{tabular}

CI, confidence interval.

A

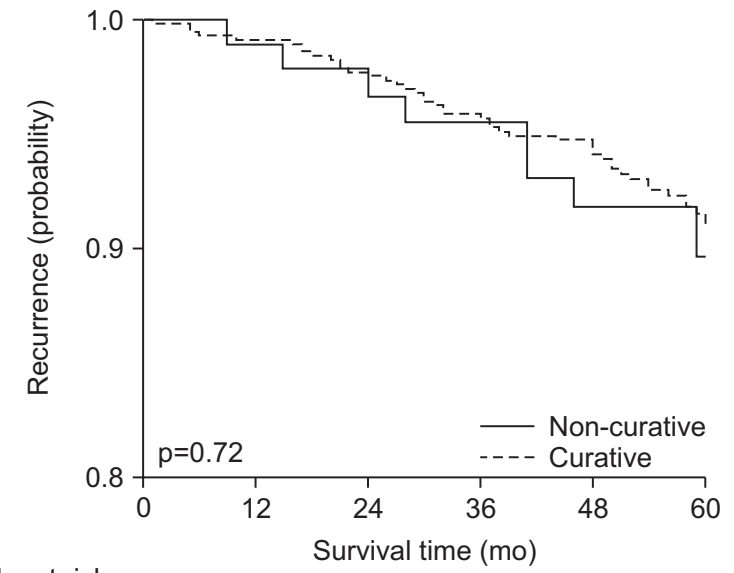

No. at risk

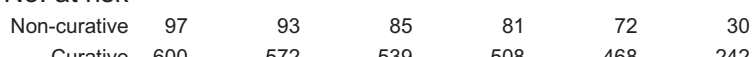

B

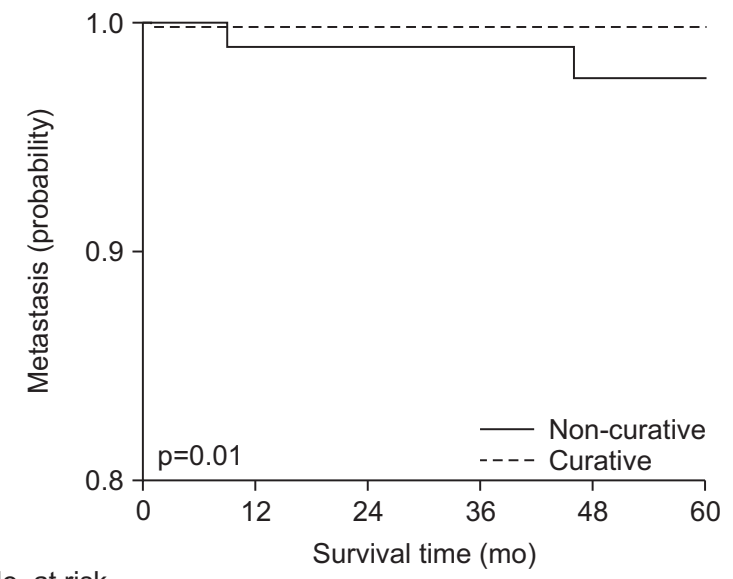

No. at risk

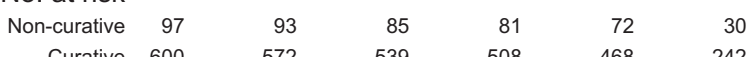

D

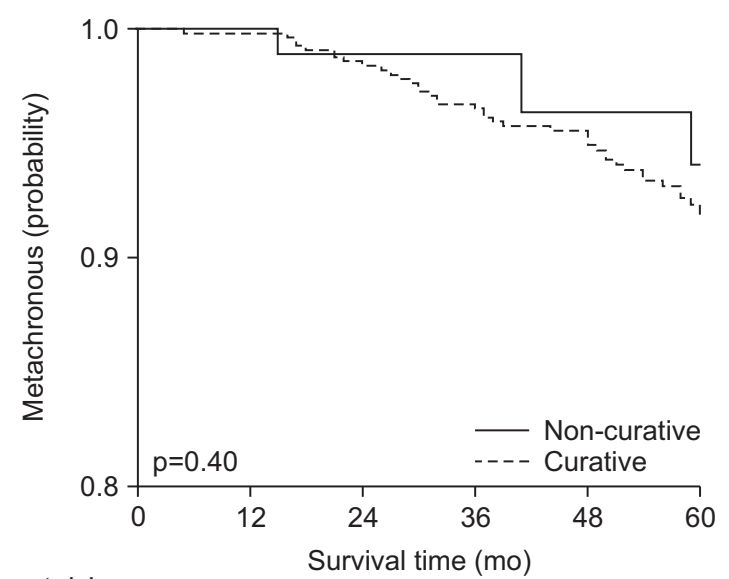

No. at risk

$\begin{array}{lllllll}\text { Non-curative } & 97 & 93 & 85 & 81 & 72 & 30\end{array}$ $\begin{array}{lrrrrr}\text { Curative } & 600 & 572 & 539 & 508 & 468\end{array}$

No. at risk

$\begin{array}{lllllll}\text { Non-curative } & 97 & 93 & 85 & 81 & 72 & 30\end{array}$

$\begin{array}{llllll}600 & 572 & 539 & 508 & 468 & 242\end{array}$

Fig. 4. Recurrence rates according to curative resection. (A) All recurrences of gastric cancer, including local, distant, and metachronous tumor (B) Distant metastasis. (C) Local recurrence of index cancer. (D) Metachronous recurrence. 

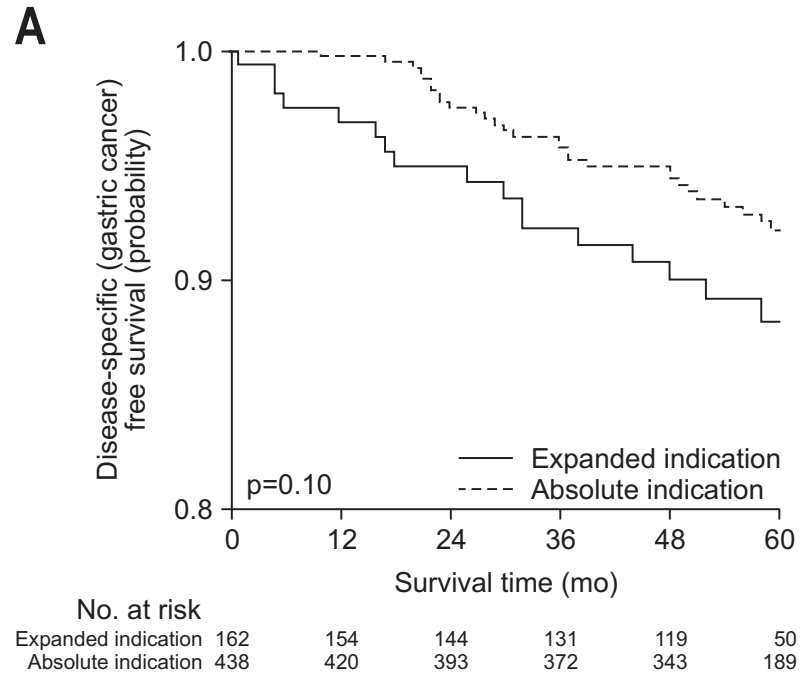

C

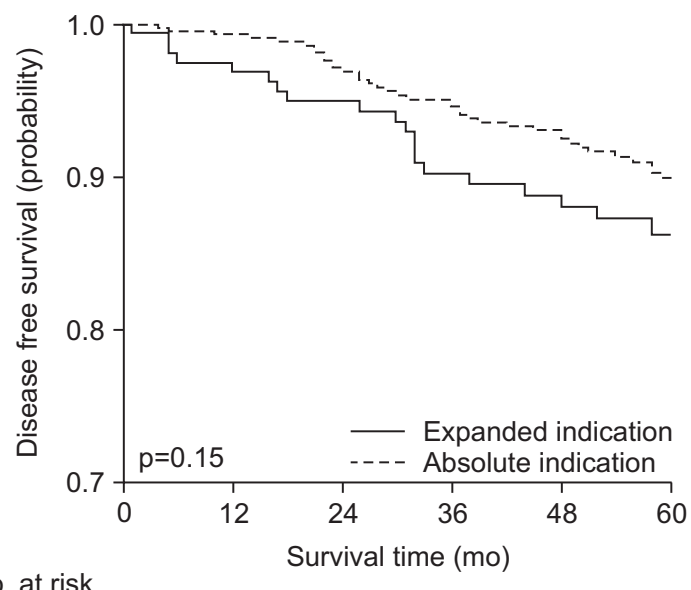

. at risk

Expanded indication 162

$\begin{array}{rrrrr}154 & 144 & 131 & 119 & 50 \\ 420 & 393 & 372 & 343 & 189\end{array}$

Fig. 5. Survival rates for absolute and expanded criteria.

87.9\% (95\% CI, 85.3\% to 90.5\%) (Table 2, Fig. 2). Although 5-year disease specific free survival rate and 5-year disease specific survival rate were not different between curative and noncurative group, 5-year overall survival rate and 5-year diseasefree survival rate were significantly higher in curative group.

All survival rates analyzed in primary and secondary outcomes were not significantly inferior that of surgery. ${ }^{14}$

\section{Survival rates by subgroup analysis}

In subgroup analysis, survival rates were compared according to curative resection and criteria in final pathological diagnosis. Five-year disease specific free survival and 5-year disease specific survival rate were not significantly different between curative and non-curative group, but 5-year overall survival and 5-year disease-free survival rate were significantly higher in curative group (Fig. 3). In recurrence rate, overall, local and metachronous recurrence rate were not different between curative and non-curative resection group, but the rate of distant metastasis was significantly higher in non-curative resection
B

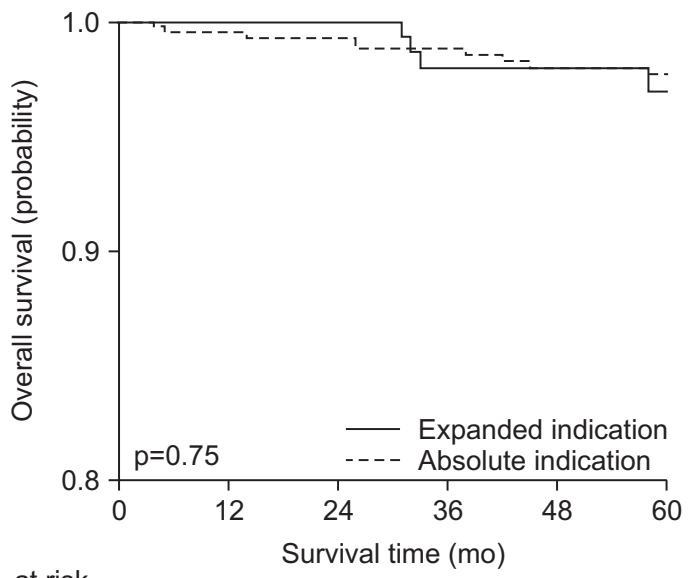

No. at risk

$\begin{array}{lllllr}\text { Expanded indication } 162 & 158 & 152 & 143 & 131 & 56 \\ \text { Absolute indication } & 438 & 421 & 402 & 386 & 359\end{array}$

D

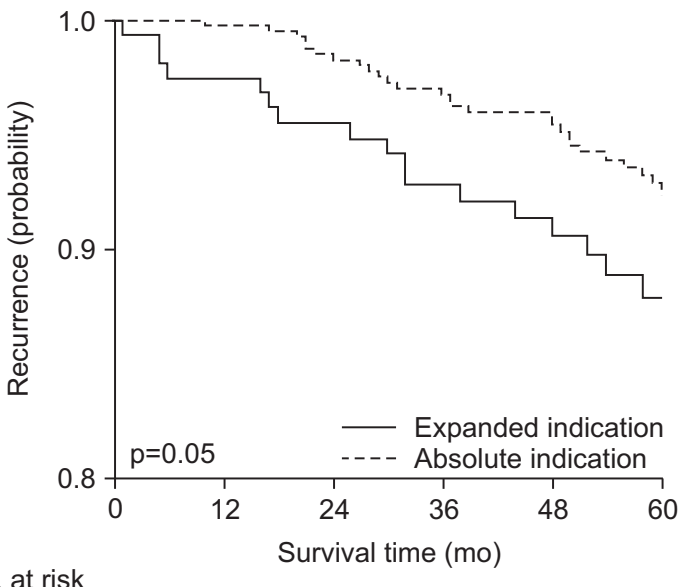

No. at risk

Expanded indication 162

$\begin{array}{rrrrr}153 & 143 & 132 & 120 & 50 \\ 419 & 396 & 376 & 348 & 192\end{array}$

group (Table 3, Fig. 4).

All survival rates analyzed in primary and secondary outcomes were not significantly different between the groups by absolute and expanded criteria (Fig. 5).

\section{Clinical course of non-curative and recurrent cases}

A total of 97 patients did not achieved initial curative resection. Of these patients, 42 patients (43.8\%) had received additional surgical resection. All recurrence rate including local, distant, and metachronous recurrence was 9.0\% (Table 3). Local and distant tumor had recurred in two patients during followup, respectively, in whom 1 patient had died of recurred cancer.

In curative group, local and metachronous recurrence were found in $0.7 \%$ and $8.1 \%$ during follow-up, respectively, who were treated by additional endoscopic or surgical resection.

\section{DISCUSSION}

In this prospective cohort study, the long-term clinical out- 
comes of ESD for EGC were evaluated by nationwide enrollment and follow-up in multicenter in Korea. Although several studies have reported the long-term clinical outcomes of ESD for EGC, the previous reports had the limitations of single center study, mostly retrospective manner, frequent loss of follow-up, heterogenous criteria of enrollment, various follow-up and treatment strategy of recurrence, and lack of unified pathological review. In this study, the criteria of enrollment was similar to absolute indication except gross tumor size of $3 \mathrm{~cm}$. Therefore, most patients had showed the final pathological result within expanded criteria, which could maintain homogeneity of risk of recurrence during long-term follow-up. Serial follow-up with endoscopy and abdominal CT was unified for detection of local or distant recurrence, which had the strength of completeness in the evaluation of long-term clinical outcomes. Moreover, central pathological review has eliminated the inter-observer variations in the histological diagnosis.

All the survival rates including 5-year disease specific free survival as primary outcome in curative group were not significantly inferior to surgery, which shows that ESD has an excellent curative result and can be accepted as a standard treatment for EGC within expanded criteria. Local recurrence had developed in $0.7 \%$ during follow-up of curative group. Although complete resection was confirmed by pathological mapping, false negativity could occur because minute tumor could exist in the margin between the sections, and skipped minute tumor beyond the resection could grow and recur at the site of ESD during follow-up.

Metachronous tumor had developed in 7.8\% of the patients during follow-up. Contrary to surgical resection, ESD can preserve stomach totally, and the remained gastric mucosa has the higher risk of carcinogenesis than that of normal healthy controls. The rate of metachronous tumor development has reported to be $4 \%$ to $10 \%$ during 5 -year follow-up in the previous studies, which is not different from this result.

Lymph node or distant metastasis was found in $0.2 \%$ of curative group, which was not different from previous reports. Longterm prognosis is usually influenced by lymph node or distant metastasis rather than local or metachronous recurrence. Although local or metachronous recurrence can be usually treated by additional endoscopic or surgical resection, lymph node or distant metastasis may not be cured by local treatment, and can influence on disease-specific survival. As ESD cannot remove perigastric lymph nodes but the tumor itself in the stomach, the prediction of the risk of lymph node and distant metastasis is mandatory prior to ESD. The lesions within expanded criteria have showed the negligible risk of lymph node and distant metastasis in previous reports. However, several studies have reported the cases of lymph node or distant metastasis during follow-up even after curative resection of EGC within expanded criteria. Although the rate of recurrence in expanded criteria was minimal and not different from this result, the previous studies showed the lymph node or distant metastasis could occur even in expanded criteria. Therefore, serial surveillance is mandatory for the detection of lymph node or distant metastasis after ESD for EGC.

Five-year disease specific free survival was achieved in $90.6 \%$, which means that the recurrence or death from index cancer had occurred in 9.4\% during follow-up. Five-year overall survival rate was $96.6 \%$, which was not inferior to that of surgery. Furthermore, 5-year disease specific survival had showed 99.8\%, which showed that death from index cancer was negligible even in cases with recurrence and ESD had excellent prognosis of EGC.

Five-year disease specific free survival and 5-year disease specific survival rate were not significantly different between curative and non-curative group, which showed that noncurative resection might not increase recurrence or death from index cancer. Local recurrence is usually associated with completeness of resection, not depth of tumor invasion or differentiation. Although the non-curative group had the higher risk of lymph node or distant metastasis, the rate was too low to show the difference between the groups. Five-year overall survival and 5-year disease-free survival rate were significantly higher in curative group, which might mean that all death might be higher in non-curative group by additional treatment including surgery or other serious diseases which could not be adequately managed.

All survival rates were not significantly different according to criteria. Expanded criteria as well as absolute indication has reported to have minimal risk of local recurrence or distant metastasis, and showed excellent long-term survival. As metachronous tumor development was not usually associated with the stage of index tumor, the rate was not different between the groups.

Local recurrence and metachronous tumor were mainly treated with additional surgical or endoscopic resection, and the prognosis was excellent. Although cure was not achieved in some cases with distant metastasis, the proportion was relatively very low.

Our study has several advantages. First, this was a nationwide multicenter prospective cohort study, and showed high followup rate without significant loss. Also, selection and recall bias were minimized by prospective manner. Second, the pathological evaluation for ESD specimens was performed by an independent central review board specialized in gastrointestinal tumors, and inter-observer variations could be compromised.

The limitations of this study are that the results may overestimate the long-term clinical outcomes of ESD for EGC and not be applied generally because the endoscopists were skillful experts in high-volume centers.

In conclusion, this prospective cohort study confirmed the excellent long-term clinical outcomes of ESD for EGC within expanded as well as absolute criteria. From this result, ESD is 
expected to be a standard treatment for EGC in expanded criteria in final pathology with comparable clinical outcomes by surgical treatment.

\section{CONFLICTS OF INTEREST}

No potential conflict of interest relevant to this article was reported.

\section{ACKNOWLEDGEMENTS}

This study was conducted as part of project number NA2010001 funded by the National Evidence-based Healthcare Collaborating Agency (NECA) in Korea and this work was funded by the National Evidence-based Healthcare Collaborating Agency (NECA), project number (NA 16-001).

\section{REFERENCES}

1. Karimi P, Islami F, Anandasabapathy S, Freedman ND, Kamangar F. Gastric cancer: descriptive epidemiology, risk factors, screening, and prevention. Cancer Epidemiol Biomarkers Prev 2014;23:700713.

2. Jung KW, Won YJ, Oh CM, et al. Cancer statistics in Korea: incidence, mortality, survival, and prevalence in 2014. Cancer Res Treat 2017;49:292-305.

3. Suh M, Choi KS, Lee YY, Jun JK. Trends in cancer screening rates among Korean men and women: results from the Korean National Cancer Screening Survey, 2004-2012. Cancer Res Treat 2013;45:86-94.

4. Kim YG, Kong SH, Oh SY, et al. Effects of screening on gastric cancer management: comparative analysis of the results in 2006 and in 2011. J Gastric Cancer 2014;14:129-134.
5. Kim SG, Ji SM, Lee NR, et al. Quality of Life after endoscopic submucosal dissection for early gastric cancer: a prospective multicenter cohort study. Gut Liver 2017;11:87-92.

6. Choi IJ, Lee NR, Kim SG, et al. Short-term outcomes of endoscopic submucosal dissection in patients with early gastric cancer: a prospective multicenter cohort study. Gut Liver 2016;10:739-748.

7. Choi J, Kim SG, Im JP, Kim JS, Jung HC. Long-term clinical outcomes of endoscopic resection for early gastric cancer. Surg Endosc 2015;29:1223-1230.

8. Choi MK, Kim GH, Park DY, et al. Long-term outcomes of endoscopic submucosal dissection for early gastric cancer: a singlecenter experience. Surg Endosc 2013;27:4250-4258.

9. Choi IJ, Lee JH, Kim YI, et al. Long-term outcome comparison of endoscopic resection and surgery in early gastric cancer meeting the absolute indication for endoscopic resection. Gastrointest Endosc 2015;81:333-341.e1.

10. Schlemper RJ, Riddell RH, Kato Y, et al. The Vienna classification of gastrointestinal epithelial neoplasia. Gut 2000;47:251-255.

11. Yoon H, Kim SG, Choi J, et al. Risk factors of residual or recurrent tumor in patients with a tumor-positive resection margin after endoscopic resection of early gastric cancer. Surg Endosc 2013;27:1561-1568.

12. Japanese Gastric Cancer Association. Japanese gastric cancer treatment guidelines 2010 (ver. 3). Gastric Cancer 2011;14:113123.

13. Kim Y, Kim YW, Choi IJ, et al. Cost comparison between surgical treatments and endoscopic submucosal dissection in patients with early gastric cancer in Korea. Gut Liver 2015;9:174-180.

14. Park SR, Kim MJ, Ryu KW, et al. Prognostic value of preoperative clinical staging assessed by computed tomography in resectable gastric cancer patients: a viewpoint in the era of preoperative treatment. Ann Surg 2010;251:428-435. 